though it always happens with the models they have calculated, Landsberg and Park cannot prove that it must happen.

Such behaviour could explain why our Universe seems to be so close to the dividing line between continued infinite expansion and just turning back into collapse at some future time. In the Landsberg-Park cosmology, successive cycles of expansion/collapse bring the model ever closer to this dividing line, and the closeness of our Universe to the "just bound" condition might suggest that it has already been through many cycles. So, like the best theories, this one makes a firm prediction: future observations should show that the Universe is indeed just bound, and not just unbound.

But there are two snags with the theory. First, if successive cycles expand to bigger and bigger radii, running the calculation backwards would suggest that the bounce itself started in some infinitesimal hiccup some large number of cycles ago, so we still have an origin problem but in a different form. Second, and perhaps more serious, if the closeness of the Universe to the "just bound" condition means that it has been through many cycles then there has been ample opportunity for entropy to increase following the constant thermodynamic arrow of time of the model. Yet the most obvious feature of our Universe is its low entropy; as Hermann Bondi has said "thermodynamic properties tend to be very deep and significant: the fact that our night sky is very black, with very bright points, the stars, in it, may be the profoundest piece of knowledge of the universe that we have". But these, of course, are just the kind of questions that a more sophisticated version of this simple model might be expected to tackle.

\section{Rotation of planetary atmospheres}

from a Correspondent

A meeting of the Royal Astronomical Society, Institute of Physics and the Royal Meteorological Society, held in London on November 28, 1975 , provided interdisciplinary discussions of the atmospheric motions at all atmospheric levels.

STudres of atmospheric motions concern scientists from various backgrounds, whose research interests generally confine them to a small region of the entire atmosphere. Meteorologists, for example, are concerned with the lowest levels which constitute the troposphere and stratosphere which extend from the surface to about $35 \mathrm{~km}$ for their studies of weather and climate; aeronomers are interested in the physics, chemistry and motions of the remaining portions of the neutral atmosphere, the stratosphere, mesophere and thermosphere, which extend to about $100 \mathrm{~km}$ above the surface; while a third group study the ionised layers of the upper atmosphere. It may be scientifically convenient to partition the atmosphere in this way according to the dominant physical processes that occur. It is, however, of fundamental importance that we now study the whole atmosphere, and consider the energy transfers between these regions, so that we may determine whether external effects such as solar activity or stratospheric compositional changes could have any effect upon our climate in the troposphere.

By tracking orbiting satellites for more than a decade, D. King-Hele (Royal Aircraft Establishment, Farnborough) has found that Earth's atmosphere above about $100 \mathrm{~km}$ moves faster than the solid body of the planet. This is called "super rotation". The magnitude of the motion is highly variable, with altitude, latitude and time, although the largest value measured so far is about $160 \mathrm{~m} \mathrm{~s}^{-1}$ at an altitude of $240 \mathrm{~km}$ from tracking the COSMOS 344 satellite. The normal rotation at these levels would imply motions of $100 \mathrm{~m} \mathrm{~s}^{-1}$. The Explorer 1 satellite has been tracked through two solar maxima, and suggested that the rotation rate decreased with a corresponding decrease in solar activity. Subrotational motions $\left(\leqslant 100 \mathrm{~m} \mathrm{~s}^{-1}\right)$ have so far been found only in the afternoon period at these altitudes. H. Rishbeth (SRC Appleton Laboratory, Slough) indicated that there is inadequate information to construct a theoretical model in an attempt to explain these motions. Unlike the winds in the lower atmosphere, they were not in geostrophic balance at these levels. $\mathrm{He}$ indicated that the driving mechanisms would differ on a local basis (for example, the polar cap, auroral zone, equatorial latitudes) and an integrated measurement/theoretical programme was urgently required. The deposition of interplanetary matter, in particular meteoroids, has often been thought to be a possible additional heating source of the upper atmosphere. D. W. Hughes (University of Sheffield) showed however, that there is insufficient material to modify the upper atmospheric motions in any significant manner.

In the stratosphere, wave motions play a major role in modifying the atmospheric motions as discussed by
D. G. Andrews (University College, London; Meteorological Office). The equatorial region has been found to undergo a quasi-biennial oscillation (QBO) in which, in a period of about 26 months, easterly motions become reversed into westerly motions. $\mathrm{He}$ suggested that Kelvin waves with a 15-d period could account for the easterly flow, while a Rossby gravity wave with a 4-d period could be responsible for the westerly flow. Andrews also thought that tropospheric changes may influence these stratospheric motions through the vertical propagation of energy. A. White (Imperial College, London) discussed the importance of baroclinic eddies in the transfer of troposphere energy. $\mathrm{He}$ showed that the structure of the flow was sensitive to the pole-to-equator temperature gradient and that changes in this parameter would modify the flow, producing a different climatology. White's studies indicated that simple models may be constructed to study climatic change over time intervals of several decades, through the parameterisation of the heat transfer effects of these eddies.

Other planetary atmospheres exhibit super rotation features, whose understanding could assist in determining the dominant driving mechanisms of the corresponding features of the Earth's atmosphere. R. A. Plumb (Meteorological Office) discussed the rotation of the Venusian stratosphere which moves sixty times faster than the solid body of the planet, producing zonal winds of about $100 \mathrm{~m} \mathrm{~s}^{-1}$. The motion is generated by the upward momentum transport by the thermal tide in the Venusian stratosphere, driven by solar energy absorbed by the opaque clouds whose tops occur at about $200 \mathrm{mbar}$. Similar thermal forcing could not occur in the Earth's atmosphere, since most of the solar radiation is absorbed at the surface. Jupiter and Saturn also exhibit equatorial jets with zonal velocities of about 100 and $400 \mathrm{~m} \mathrm{~s}^{-1}$ respectively. R. Hide (Meteorological Office) stated that currently available theories were unable to account for these features.

This meeting emphasised the importance of the transfer of energy between atmospheric regions in explaining many of the observed motions. A more detailed understanding of the atmospheric dynamics requires further observations at all levels, which for the Earth's atmosphere is possible through the presently planned satellite and rocket programme as D. Rees (University College London) described. We may then expect further tripartite meetings of this type which are an invaluable forum for discussing the motions of planetary atmospheres. 